\title{
Homeomorphism Mapping Based Neural Networks for Finite Time Constraint Control of a Class of Nonaffine Pure-Feedback Nonlinear Systems
}

\author{
Jianhua Zhang $\mathbb{D}^{1,2}$ Quanmin Zhu $\mathbb{D}^{3},{ }^{3}$ Yang Li $\mathbb{D}^{1,2}$ and Xueli Wu ${ }^{1,2}$ \\ ${ }^{1}$ Hebei University of Science and Technology, Shijiazhuang, Hebei 050018, China \\ ${ }^{2}$ Hebei Provincial Research Center for Technologies in Process Engineering Automation, Shijiazhuang, Hebei 050018, China \\ ${ }^{3}$ Department of Engineering Design and Mathematics, University of the West of England, Coldharbour Lane, Bristol BS16 1QY, UK \\ Correspondence should be addressed to Jianhua Zhang; jianhuazhang@aliyun.com
}

Received 15 February 2019; Revised 2 April 2019; Accepted 24 April 2019; Published 13 May 2019

Guest Editor: Chun Wei

Copyright (C) 2019 Jianhua Zhang et al. This is an open access article distributed under the Creative Commons Attribution License, which permits unrestricted use, distribution, and reproduction in any medium, provided the original work is properly cited.

\begin{abstract}
This paper proposes a new scheme for solving finite time neural networks adaptive tracking control issue for the nonaffine purefeedback nonlinear system. The procedure, based on homeomorphism mapping and backstepping, effectively deals with constraint control and design difficulty induced by pure-feedback structure. The most outstanding novelty is that finite time adaptive law is proposed for training weights of neural networks. Furthermore, by combining finite time adaptive law and Lyapunov-based arguments, a valid finite time adaptive neural networks controller design algorithm is presented to ensure that system is practical finite stable (PFS) rather than uniformly ultimately bounded (UUB). Because of using the finite time adaptive law to training weights of neural networks, the closed-loop error system signals are in assurance of bounded in finite time. Benchmark simulations have well demonstrated effectiveness and efficiency of the proposed approach.
\end{abstract}

\section{Introduction}

During the past decades, lots of studies have been done to solve neural networks (NNs) control and improve the adaptive law for training neural networks weights [1-3]. Among these works, the core is NNs which are used as online approximation functions for the unknown nonlinearities, due to their inherent approximation capabilities $[4,5]$. Almost all the neural adaptive control designs and stability analyses are Lyapunov uniformly ultimately bounded (UUB) results [5]; based on the Krasovskii-LaSalle invariance principle, it is challenging to establish a generalized powerful framework for neural control [6-8], even though it has been used to get sufficient conditions for smooth stabilization for closed-loop systems [9-11].

Traditionally, Quadratic Lyapunov Functions are in common used to construct for analysis and control design of linear and nonlinear even time delay dynamic systems [12, 13]. There also exist some other formats of Lyapunov functions, such as integral Lyapunov function [14], barrier Lyapunov function $[15,16]$, and vector Lyapunov functions $[17,18]$. Such attempts have enhanced Lyapunov function applications in control system design [19], for example, integral Lyapunov function for controller singularity problems and barrier Lyapunov function for output constraint problems. Nonlinear systems differ from linear system, because the complexity of the relation between states and input and therefore numerical techniques (open solutions) have played a significant role in controller design process [20-22]. Young's Inequality as an important lemma has been widespread used to realize UUB (uniformly ultimately bounded) in neural network based adaptive control [23].

In terms of complex of system structure such as purefeedback and nonaffine, mean value theorem [24] is popular to use to eliminate. Due to its universal approximation capability for nonlinear systems, neural network has been extensively utilized to achieve modelling. Many elegant model structure design techniques, such as strict-feedback, 
pure-feedback, affine form, and nonaffine systems [3, 25, 26], have been obtained based on backstepping procedure and Lyapunov synthesis.

Based on virtual control and iterative learning control [25], backstepping procedure provided an important methodology to control affine or nonaffine, strict-feedback, or pure-feedback form SISO nonlinear uncertain systems, which are split into several subsystems. In every subsystem, neural networks approximate the unknown nonlinear systems with adaptive law, based on Lyapunov stability theory, then the systems achieve the asymptotic stability or uniformly ultimately bounded stable. In order to better exploit the application of adaptive neural networks [27, 28], finite time stable results are more meaningful for uncertain system $[29,30]$, high order stochastic nonlinear system [31], and interconnected nonlinear system [32].

It should be noted that the research on finite time neural networks control is still in a very beginning stage. Although there exist a few of adaptive finite control algorithms which have been developed for some special systems and bench tested with simulations and applications [33-35], it is very hard to extend the research results to the general systems or algorithms. The key issue is how to systematically obtain finite time adaptive law of neural networks $[9,36]$ weight from finite time convergence of close loop systems [37]. In regard to neural network based such adaptive control to the authors' best knowledge, there are a few results about finite time adaptive neural networks control. That is because it is not easy to design the finite time neural networks adaptive controller and lack of relevant inequality skills to finish finite time stability analysis. There has been any reference to show finite time adaptive algorithm for weights of NNs having been expanded to solve the problems of finite control for purefeedback nonaffine nonlinear systems.

Motivated by the above critical analyses, we investigated neural backstepping control via finite time adaptive algorithm. At the first stage, the homeomorphism mapping is proposed in place of barrier Lyapunov functions and commonly utilized in traditional constraints control. Then with finite adaptive algorithm, adaptive neural networks backstepping control procedure is designed for nonaffine pure-feedback nonlinear systems. Sufficient condition is given via Lyapunov finite time stability theory to make sure all the signals in the closed-loop system are global uniformly bounded in finite time and the tracking error is remained in a bounded compact set. The main contributions of this paper can be summarized as follows:

(1) This paper is the first study to propose the finite time adaptive law based on Young's inequality skill for adaptive neural networks control. The main advantages are that adaptive neural networks controls achieve finite time stabilization including weights of neural networks and closed-loop control system uniformly ultimately bounded stable in finite time.

(2) To keep full state constraints in a bounded compact set, the homeomorphism mapping is proposed in place of barrier Lyapunov functions and commonly utilized in traditional constraints control.
(3) New finite time adaptive neural networks techniques, including virtual control and finite adaptive law, are introduced for ensuring the successful design of the finite time adaptive neural networks backstepping control.

The rest of this paper is organized as follows. Section 2 gives problem formulation and preliminaries, including some definitions and necessary inequality with lemmas. Nonaffine pure-feedback nonlinear systems finite time adaptive neural networks control is considered in Section 3, and a new finite time adaptive law is developed for training neural networks. Lyapunov finite time stable theory is used to guarantee the closed-loop system signals with prescribed performance in finite time. Simulation study is presented in Section 4 to indicate efficiency and effectiveness of the procedure. The conclusion and future works are discussed in Section 5.

\section{Problem Description and Preliminaries}

In this section, problem description, the notation, basic definitions, and lemmas on the system stability are presented. Consider the following nonlinear system:

$$
\begin{aligned}
& \dot{\xi}(t)=\varphi(\xi(t), u(t), t), \quad t \geq 0 \\
& \xi(t)=\phi(t), \quad t=0 \\
& \zeta(t)=\psi(\xi(t), u(t), t)
\end{aligned}
$$

where $\xi=\left(\begin{array}{llll}\xi_{1} & \xi_{2} & \cdots & \xi_{n}\end{array}\right)^{T} \in \mathfrak{R}^{n}, u \in \Re, \zeta \in \Re^{m}, \phi(t)$ are state variable, control input, output, and initial condition, respectively, and $\varphi(\cdot), \psi(\cdot)$ are nonlinear smooth functions.

The ideal output is $\zeta_{d}(t)$; therefore, the objective is to design an adaptive state feedback to ensure the output $\zeta(t)$ tracking $\zeta_{d}(t)$ and controller in the form

$$
\begin{aligned}
& u(t)=\alpha(\xi(t), \hat{\theta}(t)) \\
& \dot{\hat{\theta}}(t)=\beta(\xi(t), \widehat{\theta}(t))
\end{aligned}
$$

where $\widehat{\theta}$ indicate the estimated values of unknown parameters and the $\theta^{*}$ indicate the ideal values. For system (1) with the homeomorphism mapping,

$$
H:\left(\underline{a}_{1} \quad \bar{a}_{1}\right) \times\left(\underline{a}_{2} \quad \bar{a}_{2}\right) \times \cdots \times\left(\underline{a}_{n} \quad \bar{a}_{n}\right) \longrightarrow \mathfrak{R}^{n}
$$

propose to transform the system to realize the constraints control, such as $x=H(\xi)$, and $\xi \in\left(\underline{a}_{1} \bar{a}_{1}\right) \times\left(\begin{array}{ll}a_{2} & \bar{a}_{2}\end{array}\right) \times \cdots \times$ $\left(\underline{a}_{n} \bar{a}_{n}\right), x \in \mathfrak{R}^{n}$, and then system (1) changes into

$$
\begin{aligned}
& \dot{x}(t)=f(x(t), u(t), t), \quad t \geq 0 \\
& x(t)=\varsigma(t), \quad t=0 \\
& y(t)=g(x(t), u(t), t)
\end{aligned}
$$

where $x=\left(\begin{array}{llll}x_{1} & x_{2} & \cdots & x_{n}\end{array}\right)^{T} \in \mathfrak{R}^{n}, u \in \mathfrak{R}, y \in \mathfrak{R}$, $\varsigma(t)$ indicate the state, control, output, and initial condition, respectively, and $f(\cdot), g(\cdot)$ are nonlinear smooth functions. 
For the homeomorphism mapping, both $x=H(\xi)$ and $\xi=$ $H^{-1}(x)$ exist, and specially, $y-y_{d}=H\left(\xi-\xi_{d}\right)$ hold.

The adaptive controller in the form

$$
\begin{aligned}
& u(t)=\alpha(x(t), \hat{\theta}(t)) \\
& \dot{\hat{\theta}}(t)=\beta(x(t), \hat{\theta}(t))
\end{aligned}
$$

and the output of system (4) $y(t)$ track the $y_{d}(t)$.

If controller (5) is designed based on system (4), then put the controller into system (1); the states $\xi$ are constraint where $\xi \in\left(\underline{a}_{1} \bar{a}_{1}\right) \times\left(\underline{a}_{2} \bar{a}_{2}\right) \times \cdots \times\left(\underline{a}_{n} \bar{a}_{n}\right)$, because the states $\xi$ and $x$ satisfy the of the homeomorphism mapping relation (3).

Remark 1. There are lots of researches about constraint control $[15,38,39]$, which employ the barrier Lyapunov functions to realize constraints. The controller design is relatively complicated because of the complexity of derivation of barrier Lyapunov functions. The homeomorphism mapping skill is easy to achieve, as the mapping is used for transformation of states, which is independent for the controller design. After that, a new model emerged, then based on the new model, all control methods can be used, such as sliding model control [40], adaptive control [26, 41], and especially neural networks control which applied without model information. Such homeomorphism mapping is easy to choose as

$$
\begin{aligned}
& \xi=\frac{2 a}{\pi} \arctan (x), \\
& \xi=b \tanh (x), \\
& \xi=\operatorname{sgn}(x) c\left(1-e^{-x^{2}}\right)^{1 / 2}
\end{aligned}
$$

which satisfy $x \in \Re$ and $|\xi|<a, b, c$, respectively.

A continuous nonaffine pure-feedback nonlinear system can be described as follows:

$$
\begin{aligned}
& \dot{\xi}_{i}=\varphi_{i}\left(\bar{\xi}_{i+1}\right), \quad 1 \leq i \leq n-1 \\
& \dot{\xi}_{n}=\varphi_{n}\left(\bar{\xi}_{n}, u\right) \\
& \zeta=\xi_{1}
\end{aligned}
$$

where $\xi=\left(\begin{array}{llll}\xi_{1} & \xi_{2} & \cdots & \xi_{n}\end{array}\right)^{T} \in \mathfrak{R}^{n}, u \in \mathfrak{R}, \zeta \in \mathfrak{R}$, $\varphi(t)$ indicate the state, control, output, and initial condition, respectively, and $\bar{\xi}_{i}=\left[\xi_{1}, \xi_{2}, \ldots, \xi_{i}\right]^{T} \in \mathfrak{R}^{i}, \varphi_{i}(\cdot)$ are smooth nonlinear functions.

Assumption 2. The nonlinear function $\varphi$ in system (1) is as follows:

$$
\begin{gathered}
\frac{\partial \varphi_{i}}{\partial \xi_{i+1}} \neq 0, \\
\frac{\partial \varphi_{n}}{\partial u} \neq 0
\end{gathered}
$$

when $1 \leq i \leq n-1$.
Based on homeomorphism mapping (7), nonlinear purefeedback nonaffine system can be described as follows:

$$
\begin{aligned}
\dot{x}_{i} & =f_{i}\left(\bar{x}_{i+1}\right), \quad 1 \leq i \leq n-1 \\
\dot{x}_{n} & =f_{n}\left(\bar{x}_{n}, u\right) \\
y & =x_{1}
\end{aligned}
$$

where $\bar{x}_{i}=\left[x_{1}, x_{2}, \ldots, x_{i}\right]^{T} \in \Re^{i}, x=\left(\begin{array}{llll}x_{1} & x_{2} & \cdots & x_{n}\end{array}\right)^{T} \in$ $\mathfrak{R}^{n}, u \in \Re, y \in \Re$, and $\varphi(t)$ indicate the state, control, output, and initial condition, respectively, and $f_{i}(\cdot)$ are nonlinear smooth functions.

Assumption 3. Nonlinear function $f_{i}$ in system (9) is as follows:

$$
\begin{aligned}
\frac{\partial f_{i}}{\partial x_{i+1}} & \neq 0, \\
\frac{\partial f_{n}}{\partial u} & \neq 0
\end{aligned}
$$

when $1 \leq i \leq n-1$.

Notation. Throughout this paper, $W \in \mathfrak{R}^{m \times n}$ represents the matrix, $W=\left[w_{i j}\right]_{m \times n}$, rational number $\eta$, and matrix, matrix $W^{\eta}$ denote element-by-element powers, $W^{\eta}=\left[w_{i j}^{\eta}\right]_{m \times n}, \widetilde{W}^{\eta T}$ denote the transposition of matrix $\widetilde{W}^{\eta}$, such as $\widetilde{W}^{\eta T}=\left(\widetilde{W}^{\eta}\right)^{T}$. $W^{*}, \widehat{W}, \widetilde{W}$ indicate ideal weight, estimated weight, and error between ideal and estimated weight.

In brief, the following radial basis function (RBF) $\mathrm{NN}$ is used to approximate the continuous function $F(x): \mathfrak{R}^{n} \longrightarrow$ $\mathfrak{R}$ over a compact set

$$
F_{N N}(x, W)=W^{T} \Psi(x)
$$

where input $x \in \Omega \subset \mathfrak{R}^{n}$, weight vector $W=$ $\left[\begin{array}{lll}w_{1} & \cdots & w_{l}\end{array}\right]^{T} \in \mathfrak{R}^{l}$, and node vector $\Psi(x)=$ $\left[\begin{array}{lll}\psi_{1}(x) & \cdots & \psi_{l}(x)\end{array}\right]^{T}$, with the element $\psi_{i}(x)$ being chosen as the commonly used Gaussian function as below

$$
\psi_{i}(x)=\exp \left[\frac{-\left(x-\mu_{i}\right)^{T}\left(x-\mu_{i}\right)}{\eta_{i}^{2}}\right], \quad i=1,2, \ldots, l .
$$

where $\mu_{i}=\left[\begin{array}{lll}\mu_{i 1} & \cdots & \mu_{i n}\end{array}\right]^{T}$, the center of the receptive field, and $\eta_{i}$ is the width of the Gaussian function.

It has been proven that RBF NN can approximate any continuous function over a compact set $\Omega_{x} \subset \mathfrak{R}^{n}$ as

$$
F(x)=W^{* T} \Psi(x)+\varepsilon(x)
$$

where $W^{*}$ is the ideal $\mathrm{NN}$ weight and $\varepsilon(x)$ is the NN approximation error.

$$
W^{*}=\arg \min _{W \in \Re^{l}}\left\{\sup \left|F(x)-W^{T} \Psi(x)\right|\right\}
$$

Definition 4. Consider system $\dot{x}=f(x, u)$, where $x$ is a state vector and $u$ is the input vector. The solution is practical finite stable (PFS) if for all $x\left(t_{0}\right)=x_{0}$, there exist $\varepsilon>0$ and $T\left(\varepsilon, x_{0}\right)<\infty$, such that $\|x\|<\varepsilon$, for all $t \geq t_{0}+T$. 
Lemma 5. Consider system $\dot{x}=f(x, u)$; suppose that there exist continuous function $V(x)$ and scalars $\lambda>0,0<\alpha<1$, and $0<\eta<\infty$ such that

$$
\dot{V}(x) \leq-\lambda V^{\alpha}(x)+\eta
$$

Then, the trajectory of system $\dot{x}=f(x, u)$ is PFS.

Proof. There exists a scalar $0<\theta \leq 1$ such that inequality (15) can be expressed as

$$
\dot{V}(x) \leq-\theta \lambda V^{\alpha}(x)-(1-\theta) \lambda V^{\alpha}(x)+\eta
$$

Clearly, $\dot{V}(x) \leq-\theta \lambda V^{\alpha}(x)$ if $V^{\alpha}(x)>\eta / \lambda(1-\theta)$. According to Lemma 5, the decrease of $V(x)$ in finite time drives the trajectories of the closed-loop system into $V^{\alpha}(x) \leq \eta / \lambda(1-$ $\theta)$. Therefore, the trajectories of the closed-loop system are bounded in finite time as

$$
\lim _{\theta \rightarrow \theta_{0}} x \in\left(V^{\alpha}(x) \leq \frac{\eta}{\lambda(1-\theta)}\right)
$$

where $0<\theta_{0}<1$. And the time needed to reach (17) is bounded as

$$
T \leq \frac{V^{1-\alpha}\left(x_{0}\right)}{\lambda \theta_{0}(1-\alpha)}
$$

where $V\left(x_{0}\right)$ is the initial value of $V(x)$.

Lemma 6 ([42], Young's inequality). For any constant $a, b \in$ $\mathfrak{R}$, the following inequality holds:

$$
a b \leq \frac{1}{p} a^{p}+\frac{1}{q} b^{q}
$$

where $p>1, q>1$, and $1 / p+1 / q=1$.

Remark 7. Based on Young's inequality, then the following inequalities hold:

$$
\begin{aligned}
z \varepsilon & \leq \frac{3}{4} z^{4 / 3}+\frac{1}{4} \varepsilon^{4} \\
-\widetilde{W}^{T} \widehat{W}^{1 / 3} & \leq-\frac{1}{2} \widetilde{W}^{2 / 3^{T}} \widetilde{W}^{2 / 3}+W^{* 2 / 3^{T}} W^{* 2 / 3}
\end{aligned}
$$

There are two key inequalities in adaptive neural networks finite time control.

\section{Control Design and Stability Analysis}

Consider system (9), using the coordinate transform as

$$
\begin{aligned}
z_{1} & =x_{1}-y_{d} \\
z_{i} & =x_{i}-\alpha_{i-1}, \quad i=2,3, \ldots, n \\
z_{n+1} & =u-\alpha_{n}
\end{aligned}
$$

where $\alpha_{i}$ is the virtual control and the ideal virtual control are

$$
\begin{aligned}
& \alpha_{1}^{*}=-k_{1} z_{1}^{1 / 3}+x_{2}-f_{1}+\dot{y}_{d} \\
& \alpha_{i}^{*}=-z_{i-1}-k_{i} z_{i}^{1 / 3}+x_{i+1}-f_{i}+\dot{\alpha}_{i-1}^{*} \quad 2 \leq i \leq n-1 \\
& \alpha_{n}^{*}=-z_{n-1}-k_{n} z_{n}^{1 / 3}+u-f_{n}+\dot{\alpha}_{n-1}^{*}
\end{aligned}
$$

In the first step, consider system

$$
z_{1}=x_{1}-y_{d}
$$

Then it has

$$
\dot{z}_{1}=f_{1}\left(\bar{x}_{2}\right)-\dot{y}_{d}
$$

Based on ideal virtual control law (23), choose the NN to approximate the nonlinear system $f_{1}, \bar{x}_{2} \in \Omega_{1} \subset \mathfrak{R}^{2}$, and $\Omega_{1}$ is compact set

$$
f_{1}\left(\bar{x}_{2}\right)-\dot{y}_{d}=W_{1}^{T *} \Psi_{1}\left(\bar{x}_{2}\right)+\varepsilon_{1}
$$

Therefore

$$
\dot{z}_{1}=W_{1}^{* T} \Psi_{1}\left(\bar{x}_{2}\right)+\varepsilon_{1}
$$

Choose the practical virtual control law

$$
\alpha_{1}=-k_{1} z_{1}^{1 / 3}+x_{2}-\widehat{W}_{1}^{T} \Psi_{1}\left(\bar{x}_{2}\right)
$$

Choose the adaptive law

$$
\dot{\widehat{W}}_{1}=\Gamma_{1}\left[z_{1} \Psi_{1}\left(\bar{x}_{2}(t)\right)-\sigma_{1} \widehat{W}_{1}^{1 / 3}\right]
$$

where $\Gamma_{1}=\Gamma_{1}^{T}>0$ and $\sigma_{1}>0$ is positive constant design parameters.

Then based on (27) and (28)

$$
\begin{aligned}
\dot{z}_{1}= & W_{1}^{* T} \Psi_{1}\left(\bar{x}_{2}\right)+\varepsilon_{1}-k_{1} z_{1}^{1 / 3}+x_{2}-\alpha_{1} \\
& -\widehat{W}_{1}^{T} \Psi_{1}\left(\bar{x}_{2}\right)
\end{aligned}
$$

let

$$
z_{2}=x_{2}-\alpha_{1}
$$

therefore

$$
\dot{z}_{1}=-\widetilde{W}_{1}^{T} \Psi_{1}\left(\bar{x}_{2}\right)-k_{1} z_{1}^{1 / 3}+z_{2}+\varepsilon_{1}
$$

where

$$
\widetilde{W}_{1}=\widehat{W}_{1}-W_{1}^{*}
$$

Choose Lyapunov candidate function

$$
V_{1}=\frac{1}{2} z_{1}^{2}+\frac{1}{2} \widetilde{W}_{1}^{\mathrm{T}} \Gamma_{1}^{-1} \widetilde{W}_{1}
$$

then

$$
\begin{aligned}
\dot{V}_{1} & =z_{1} \dot{z}_{1}+\widetilde{W}_{1}^{\mathrm{T}} \Gamma_{1}^{-1} \dot{\widetilde{W}}_{1} \\
& =-k_{1} z_{1}^{4 / 3}+z_{1} z_{2}+z_{1} \varepsilon_{1}-\sigma_{1} \widetilde{W}_{1}^{\mathrm{T}} \widehat{W}_{1}^{1 / 3}
\end{aligned}
$$

Based on inequalities from Lemma 6 and Remark 7, the following hold:

$$
z_{1} \varepsilon_{1} \leq \frac{3}{4} z_{1}^{4 / 3}+\frac{1}{4} \varepsilon_{1}^{4}
$$


and

$$
-\sigma_{1} \widetilde{W}_{1}^{T} \widehat{W}_{1}^{1 / 3} \leq-\frac{\sigma_{1}}{2} \widetilde{W}_{1}^{(2 / 3) \mathrm{T}} \widetilde{W}_{1}^{2 / 3}+\sigma_{1} W_{1}^{*(2 / 3) \mathrm{T}} W_{1}^{* 2 / 3}
$$

Then based on (35), it gives

$$
\begin{aligned}
\dot{V}_{1}= & -\left(k_{1}-\frac{3}{4}\right) z_{1}^{4 / 3}+z_{1} z_{2}-\frac{\sigma_{1}}{2} \widetilde{W}_{1}^{(2 / 3) \mathrm{T}} \widetilde{W}_{1}^{2 / 3}+\frac{1}{4} \varepsilon_{1}^{4} \\
& +\sigma_{1} W_{1}^{*(2 / 3) \mathrm{T}} W_{1}^{* 2 / 3}
\end{aligned}
$$

then it has

$$
\begin{aligned}
\dot{V}_{1} \leq & z_{1} z_{2}-\eta_{1}\left(\frac{1}{2} z_{1}^{2}\right)^{2 / 3}-\eta_{1}\left(\frac{1}{2} \widetilde{W}_{1}^{\mathrm{T}} \Gamma_{1}^{-1} \widetilde{W}_{1}\right)^{2 / 3} \\
& +\frac{1}{4} \varepsilon_{1}^{4}+\sigma_{1} W_{1}^{*(2 / 3) \mathrm{T}} W_{1}^{* 2 / 3} \\
\leq & z_{1} z_{2}-\eta_{1} V_{1}^{2 / 3}+\delta_{1}
\end{aligned}
$$

where

$$
\begin{aligned}
& \eta_{1}=\min \left(4^{1 / 3}\left(k_{1}-\frac{3}{4}\right), \frac{\sigma_{1}}{2^{1 / 3}\left(\lambda_{\max }\left(\Gamma_{1}\right)\right)^{2 / 3}}\right) \\
& \delta_{1}=\frac{1}{4} \varepsilon_{1}^{4}+\sigma_{1} W_{1}^{*(2 / 3) \mathrm{T}} W_{1}^{* 2 / 3}
\end{aligned}
$$

The $i$ th step $2 \leq i \leq n-1$

$$
z_{i}=x_{i}-\alpha_{i-1}
$$

Choose the virtual control law (23), choose the NN to approximate the nonlinear system $f_{i}\left(\bar{x}_{i+1}\right)-\dot{\alpha}_{i-1}, \bar{x}_{i+1} \in \Omega_{i} \subset$ $\mathfrak{R}^{i+1}$, and $\Omega_{i}$ is compact set

$$
f_{i}\left(\bar{x}_{i+1}(t)\right)-\dot{\alpha}_{i-1}=W_{i}^{* T} \Psi_{i}\left(\bar{x}_{i+1}\right)+\varepsilon_{i}
$$

where

$$
\dot{\alpha}_{i-1}=\sum_{j=1}^{i} \frac{\partial \alpha_{i-1}}{\partial x_{j}} \dot{x}_{j}+\sum_{j=1}^{i-1} \frac{\partial \alpha_{i-1}}{\partial \widehat{W}_{j}^{T}} \hat{\widehat{W}}_{j}^{T}+y_{d}^{(i)}
$$

Based on the system,

$$
\dot{z}_{i}=W_{i}^{* T} \Psi_{i}\left(\bar{x}_{i+1}\right)+\varepsilon_{i}
$$

Choose the practical virtual control law

$$
\alpha_{i}=-z_{i-1}-k_{i} z_{i}^{1 / 3}+x_{i+1}-\widehat{W}_{i}^{T} \Psi_{i}\left(\bar{x}_{i+1}\right)
$$

Choose the adaptive law

$$
\dot{\widehat{W}}_{i}=\Gamma_{i}\left[z_{i} \Psi_{i}\left(\bar{x}_{i+1}\right)-\sigma_{i} \widehat{W}_{i}^{1 / 3}\right]
$$

where $\Gamma_{i}=\Gamma_{i}^{T}>0$ and $\sigma_{i}>0$ is positive constant design parameters. Then

$$
\dot{z}_{i}=-\widetilde{W}_{i} \Psi_{i}\left(\bar{x}_{i+1}\right)-z_{i-1}-k_{i} z_{i}^{1 / 3}+z_{i+1}+\varepsilon_{i}
$$

where

$$
\widetilde{W}_{i}=\widehat{W}_{i}-W_{i}^{*}
$$

Choose Lyapunov candidate function

$$
V_{i}=\frac{1}{2} z_{i}^{2}+\frac{1}{2} \widetilde{W}_{i}^{\mathrm{T}} \Gamma_{i}^{-1} \widetilde{W}_{i}
$$

then

$$
\begin{aligned}
\dot{V}_{i} & =z_{i} \dot{z}_{i}+\widetilde{W}_{i} \Gamma_{i}^{-1} \dot{\widetilde{W}}_{i}^{\mathrm{T}} \\
& =-z_{i-1} z_{i}-k_{i} z_{i}^{4 / 3}+z_{i} z_{i+1}+z_{i} \varepsilon_{i}-\sigma_{i} \widetilde{W}_{i}^{\mathrm{T}} \widehat{W}_{i}^{1 / 3}
\end{aligned}
$$

Based on Lemma 6 and Remark 7, the following inequalities hold:

$$
z_{i} \varepsilon_{i} \leq \frac{3}{4} z_{i}^{4 / 3}+\frac{1}{4} \varepsilon_{i}^{4}
$$

and

$$
-\sigma_{i} \widetilde{W}_{i}^{\mathrm{T}} \widehat{W}_{i}^{1 / 3} \leq-\frac{\sigma_{i}}{2} \widetilde{W}_{i}^{(2 / 3) \mathrm{T}} \widetilde{W}_{i}^{2 / 3}+\sigma_{i} W_{i}^{*(2 / 3) \mathrm{T}} W_{i}^{* 2 / 3}
$$

Then based on (49), it yields

$$
\begin{aligned}
\dot{V}_{i}= & z_{i} \dot{z}_{i}+\widetilde{W}_{i}^{\mathrm{T}} \Gamma_{i}^{-1} \dot{\bar{W}}_{i} \\
= & -z_{i-1} z_{i}-\left(k_{i}-\frac{3}{4}\right) z_{i}^{4 / 3}+z_{i} z_{i+1} \\
& \quad-\frac{\sigma_{i}}{2} \widetilde{W}_{i}^{(2 / 3) \mathrm{T}} \widetilde{W}_{i}^{2 / 3}+\frac{1}{4} \varepsilon_{i}^{4}+\sigma_{i} W_{i}^{*(2 / 3) \mathrm{T}} W_{i}^{* 2 / 3}
\end{aligned}
$$

And

$$
\begin{aligned}
\dot{V}_{i} \leq & -z_{i-1} z_{i}+z_{i} z_{i+1}-\eta_{i}\left(\frac{1}{2} z_{i}^{2}\right)^{2 / 3} \\
& -\eta_{i}\left(\frac{1}{2} \widetilde{W}_{i}^{\mathrm{T}} \Gamma_{i}^{-1} \widetilde{W}_{i}\right)^{2 / 3}+\frac{1}{4} \varepsilon_{i}^{4} \\
& +\sigma_{i} W_{i}^{*(2 / 3) \mathrm{T}} W_{i}^{* 2 / 3} \\
\leq & -z_{i-1} z_{i}+z_{i} z_{i+1}-\eta_{i} V_{i}^{2 / 3}+\delta_{i}
\end{aligned}
$$

where

$$
\begin{aligned}
& \eta_{i}=\min \left(4^{1 / 3}\left(k_{i}-\frac{3}{4}\right), \frac{\sigma_{i}}{2^{1 / 3}\left(\lambda_{\max }\left(\Gamma_{i}\right)\right)^{2 / 3}}\right) \\
& \delta_{i}=\frac{1}{4} \varepsilon_{i}^{4}+\sigma_{i} W_{i}^{*(2 / 3) \mathrm{T}} W_{i}^{* 2 / 3}
\end{aligned}
$$

The $n$th step is the most important step. Based on the system

$$
z_{n}=x_{n}-\alpha_{n-1}
$$

Choose the NN to approximate the nonlinear system $f_{n}\left(\bar{x}_{n}, u\right)-\dot{\alpha}_{n-1}, \bar{x}_{n+1}^{T} \triangleq\left[\bar{x}_{n}^{T} u\right] \in \Omega_{n} \subset \mathfrak{R}^{n+1}$, and $\Omega_{n}$ is compact set

$$
f_{n}\left(\bar{x}_{n}, u\right)-\dot{\alpha}_{n-1}=W_{n}^{* T} \Psi_{n}\left(\bar{x}_{n+1}\right)+\varepsilon_{n}
$$


where

$$
\dot{\alpha}_{n-1}=\sum_{j=1}^{n} \frac{\partial \alpha_{n-1}}{\partial x_{j}} \dot{x}_{j}+\sum_{j=1}^{n-1} \frac{\partial \alpha_{n-1}}{\partial \widehat{\widehat{W}}_{j}^{T}} \dot{\widehat{W}}_{j}^{T}
$$

Based on system

$$
\dot{z}_{n}=W_{n}^{* T} \Psi_{n}\left(\bar{x}_{n+1}\right)+\varepsilon_{n}
$$

Choose practical virtual control law

$$
\alpha_{n}=-z_{n-1}-k_{n} z_{n}^{1 / 3}+u-\widehat{W}_{n}^{T} \Psi_{n}\left(\bar{x}_{n+1}\right)
$$

Choose adaptive law

$$
\dot{\widehat{W}}_{n}=\Gamma_{n}\left[z_{n} \Psi_{n}^{T}\left(\bar{x}_{n+1}\right)-\sigma_{n} \widehat{W}_{n}^{1 / 3}\right]
$$

where $\Gamma_{n}=\Gamma_{n}^{T}>0$ and $\sigma_{n}>0$ are positive constant design parameters, then

$$
\dot{z}_{n}=-\widetilde{W}_{n}^{T} \Psi_{n}\left(\bar{x}_{n}(t)\right)-z_{n-1}-k_{n} z_{n}^{1 / 3}+z_{n+1}+\varepsilon_{n}
$$

where

$$
\widetilde{W}_{n}=\widehat{W}_{n}-W_{n}^{*}
$$

From the inequality

$$
-\sigma_{n} \widetilde{W}_{n}^{T} \widehat{W}_{n}^{1 / 3} \leq-\frac{\sigma_{n}}{2} \widetilde{W}_{n}^{2 / 3^{T}} \widetilde{W}_{n}^{2 / 3}+\sigma_{n} W_{n}^{* 2 / 3^{T}} W_{n}^{* 2 / 3}
$$

choose Lyapunov candidate function

$$
V_{n}=\frac{1}{2} z_{n}^{2}+\frac{1}{2} \widetilde{W}_{n} \Gamma_{n}^{-1} \widetilde{W}_{n}^{\mathrm{T}}
$$

Then

$$
\begin{aligned}
\dot{V}_{n} & =z_{n} \dot{z}_{n}+\widetilde{W}_{n} \Gamma_{n}^{-1} \dot{\widetilde{W}}_{n}^{\mathrm{T}} \\
& =-z_{n-1} z_{n}-k_{n} z_{n}^{4 / 3}+z_{n} z_{n+1}+z_{n} \varepsilon_{n}-\sigma_{n} \widetilde{W}_{n}^{\mathrm{T}} \widehat{W}_{n}^{1 / 3}
\end{aligned}
$$

Based on the lemma, the following inequalities hold:

$$
z_{n} \varepsilon_{n} \leq \frac{3}{4} z_{n}^{4 / 3}+\frac{1}{4} \varepsilon_{n}^{4}
$$

and

$$
-\sigma_{n} \widetilde{W}_{n}^{\mathrm{T}} \widehat{W}_{n}^{1 / 3} \leq-\frac{\sigma_{n}}{2} \widetilde{W}_{n}^{(2 / 3) \mathrm{T}} \widetilde{W}_{n}^{2 / 3}+\sigma_{n} W_{n}^{*(2 / 3) \mathrm{T}} W_{n}^{* 2 / 3}
$$

Then, it gives

$$
\begin{aligned}
\dot{V}_{n} \leq & -z_{n-1} z_{n}-\left(k_{n}-\frac{3}{4}\right) z_{n}^{4 / 3}+z_{n} z_{n+1} \\
& -\frac{\sigma_{n}}{2} \widetilde{W}_{n}^{(2 / 3) \mathrm{T}} \widetilde{W}_{n}^{2 / 3}+\frac{1}{4} \varepsilon_{n}^{4}+\sigma_{n} W_{n}^{*(2 / 3) \mathrm{T}} W_{n}^{* 2 / 3}
\end{aligned}
$$

then

$$
\begin{aligned}
\dot{V}_{n} \leq & -z_{n-1} z_{n}+z_{n} z_{n+1}-\eta_{n}\left(\frac{1}{2} z_{n}^{2}\right)^{2 / 3} \\
& -\eta_{n}\left(\frac{1}{2} \widetilde{W}_{n}^{\mathrm{T}} \Gamma_{n}^{-1} \widetilde{W}_{n}\right)^{2 / 3}+\delta_{n} \\
\leq & -z_{n-1} z_{n}+z_{n} z_{n+1}-\eta_{n} V_{n}^{2 / 3}+\delta_{n}
\end{aligned}
$$

where

$$
\begin{aligned}
& \eta_{n}=\min \left(4^{1 / 3}\left(k_{n}-\frac{3}{4}\right), \frac{\sigma_{n}}{2^{1 / 3}\left(\lambda_{\max }\left(\Gamma_{n}\right)\right)^{2 / 3}}\right) \\
& \delta_{n}=\frac{1}{4} \varepsilon_{n}^{4}+\sigma_{n} W_{n}^{*(2 / 3) \mathrm{T}} W_{n}^{* 2 / 3}
\end{aligned}
$$

The $n+1$ th step is the most important step.

$$
z_{n+1}=u-\alpha_{n}
$$

Based on system

$$
\dot{z}_{n+1}=v-\dot{\alpha}_{n}
$$

Choose the $\mathrm{NN}$ to approximate the nonlinear system $-\dot{\alpha}_{n}\left(\bar{x}_{n+1}\right), \bar{x}_{n+1}^{T}=\left[\begin{array}{ll}\bar{x}_{n}^{T} & u\end{array}\right] \in \Omega_{n+1} \subset \mathfrak{R}^{n+1}$, and $\Omega_{n+1}$ is compact set

$$
-\dot{\alpha}_{n}=W_{n+1}^{* T} \Psi_{n+1}\left(\bar{x}_{n+1}\right)+\varepsilon_{n+1}
$$

where

$$
\dot{\alpha}_{n}=\sum_{j=1}^{n} \frac{\partial \alpha_{n}}{\partial x_{j}} \dot{x}_{j}+\sum_{j=1}^{n} \frac{\partial \alpha_{n}}{\partial \widehat{W}_{j}^{T}} \dot{\widehat{W}}_{j}^{T}+y_{d}^{(n+1)}
$$

Based on system

$$
\dot{z}_{n+1}=v+W_{n+1}^{* T} \Psi_{n+1}\left(\bar{x}_{n+1}(t)\right)+\varepsilon_{n+1}
$$

Choose adaptive law

$$
\dot{\widehat{W}}_{n+1}=\Gamma_{n+1}\left[z_{n+1} \Psi_{n+1}\left(\bar{x}_{n+1}\right)-\sigma_{n+1} \widehat{W}_{n+1}^{1 / 3}\right]
$$

where $\Gamma_{n+1}=\Gamma_{n+1}^{T}>0$ and $\sigma_{n+1}>0$ are positive constant design parameters.

$$
v=-z_{n}-k_{n+1} z_{n+1}^{1 / 3}-\widehat{W}_{n+1}^{T} \Psi_{n+1}\left(\bar{x}_{n+1}(t)\right)
$$

then

$$
\dot{z}_{n+1}=-z_{n}-k_{n+1} z_{n+1}^{1 / 3}-\widetilde{W}_{n+1}^{T} \Psi_{n+1}\left(\bar{x}_{n+1}\right)+\varepsilon_{n+1}
$$

where

$$
\widetilde{W}_{n+1}=\widehat{W}_{n+1}-W_{n+1}^{*}
$$

Choose Lyapunov candidate function

$$
V_{n+1}=\frac{1}{2} z_{n+1}^{2}+\frac{1}{2} \widetilde{W}_{n+1} \Gamma_{n+1}^{-1} \widetilde{W}_{n+1}^{\mathrm{T}}
$$


then

$$
\begin{aligned}
\dot{V}_{n+1}= & z_{n+1} \dot{z}_{n+1}+\widetilde{W}_{n+1} \Gamma_{n+1}^{-1} \dot{\widetilde{W}}_{n+1}^{\mathrm{T}} \\
= & -z_{n} z_{n+1}-k_{n+1} z_{n+1}^{4 / 3}+z_{n+1} \varepsilon_{n+1} \\
& -\sigma_{n+1} \widetilde{W}_{n+1}^{\mathrm{T}} \widehat{W}_{n+1}^{1 / 3}
\end{aligned}
$$

From the inequality

$$
\begin{aligned}
-\sigma_{n+1} \widetilde{W}_{n+1}^{T} \widehat{W}_{n+1}^{1 / 3} \leq & -\frac{\sigma_{n+1}}{2} \widetilde{W}_{n+1}{ }^{2 / 3^{T}} \widetilde{W}_{n+1}{ }^{2 / 3} \\
& +\sigma_{n+1} W_{n+1}^{*}{ }^{2 / 3^{T}} W_{n+1}^{* 2 / 3}
\end{aligned}
$$

and

$$
z_{n+1} \varepsilon_{n+1} \leq \frac{3}{4} z_{n+1}^{4 / 3}+\frac{1}{4} \varepsilon_{n+1}^{4}
$$

we have

$$
\begin{aligned}
\dot{V}_{n+1} \leq & -z_{n} z_{n+1}-\left(k_{n+1}-\frac{3}{4}\right) z_{n+1}^{4 / 3} \\
& -\frac{\sigma_{n+1}}{2} \widetilde{W}_{n+1}{ }^{2 / 3^{T}} \widetilde{W}_{n+1}^{2 / 3}+\frac{1}{4} \varepsilon_{n+1}^{4} \\
& +\sigma_{n+1} W_{n+1}^{*}{ }^{2 / 3^{T}} W_{n+1}^{* 2 / 3}
\end{aligned}
$$

Then

$$
\begin{aligned}
\dot{V}_{n+1} \leq & -z_{n} z_{n+1}-\eta_{n+1}\left(\frac{1}{2} z_{n+1}^{2}\right)^{2 / 3} \\
& -\eta_{n+1}\left(\frac{1}{2} \widetilde{W}_{n+1}^{\mathrm{T}} \Gamma_{n+1}^{-1} \widetilde{W}_{n+1}\right)^{2 / 3}+\delta_{n+1} \\
\leq & -z_{n} z_{n+1}-\eta_{n+1} V_{n+1}^{2 / 3}+\delta_{n+1}
\end{aligned}
$$

where

$$
\begin{aligned}
& \eta_{n+1} \\
& =\min \left(4^{1 / 3}\left(k_{n+1}-\frac{3}{4}\right), \frac{\sigma_{n+1}}{2^{1 / 3}\left(\lambda_{\max }\left(\Gamma_{n+1}\right)\right)^{2 / 3}}\right) \\
& \delta_{n+1}=\frac{1}{4} \varepsilon_{n+1}^{4}+\sigma_{n+1} W_{n+1}^{*}{ }^{(2 / 3) \mathrm{T}} W_{n+1}^{* 2 / 3}
\end{aligned}
$$

Choose Lyapunov candidate functions

$$
V=\sum_{i=1}^{n+1} V_{i}
$$

then based on (39), (54), (70), and (86), it has

$$
\dot{V} \leq-\eta V^{2 / 3}+\delta
$$

where

$$
\begin{aligned}
& \eta=\min \left(\eta_{i}\right), \quad i=1,2,3, \ldots, n+1 \\
& \delta=\sum_{i=1}^{n+1} \delta_{i}
\end{aligned}
$$

Theorem 8. Under Assumptions 2 and 3 and Lemmas 5 and 6, consider nonlinear system (9) for which the model dynamics is approximated by neural networks (26), (42), (57), and (74), the control law (78) with the virtual control (28), (45), and (60), and adaptive laws (29), (46), (61), and (77), then the following statements hold:

(1) All the signals of the closed-loop system, including $x_{i}, \alpha_{i}, \widehat{W}_{i}$, remain bounded all the time.

(2) The closed-loop signal $z=\left[\begin{array}{llll}z_{1} & z_{2} & \cdots & z_{n+1}\end{array}\right]^{T} \in \mathfrak{R}^{n+1}$ converges to a compact set defined by

$$
\Omega_{s}=\left\{\mathrm{z} \mid\|\mathrm{z}\|^{2} \leq \frac{\delta}{\eta}\right\}
$$

in finite time, where $\delta, \eta$ are constants related to the design parameters.

(3) The finite time $T$ is given by

$$
T \leq \frac{3 V^{1 / 3}\left(x_{0}\right)}{\eta \theta_{0}}
$$

where $\eta, \theta_{0}$ are constants related to the design parameters and $V\left(x_{0}\right)$ is constant related to Lyapunov candidate functional.

Proof. Considering Lyapunov function candidate (88) and (89), then $V$ is bounded in finite time. Therefore, it can conclude that, for all $1 \leq i \leq n+1$, the error signals $z_{i}, \widetilde{W}_{i}$ are PFS.

\section{Simulation Examples}

In this section, simulation examples show the validity and feasibility of the proposed NNs adaptive control design approach.

4.1. Mathematical Example. Consider the following dynamic system:

$$
\begin{aligned}
\dot{x}_{1}= & 0.5 \sin \left(x_{1}\right)-x_{1}+\sin \left(x_{2}\right)+x_{2}+5 \sin (t) \\
& +5 \cos (t) \\
\dot{x}_{2}= & -\sin \left(x_{2}\right)-2 x_{2}+x_{2} u+u+\sin (u) \\
y= & x_{1}
\end{aligned}
$$

where $x=\left[\begin{array}{ll}x_{1} & x_{2}\end{array}\right]^{T}, u, y$, and $y_{d}=5 \sin (t)$ is state, control input, control output, and ideal output, respectively. The control objective of the proposed design method is to make the system output $y$ follow the desired reference signal $y_{d}=5 \sin (t)$. Select the controller $\dot{u}$ and virtual control $\alpha_{1}, \alpha_{2}$ as follows:

$$
\begin{gathered}
\dot{u}=-z_{2}-k_{3} z_{3}^{1 / 3}-\widehat{W}_{3}^{T} \Psi_{3}\left(\bar{x}_{2}, u\right) \\
\alpha_{1}=-k_{1} z_{1}^{1 / 3}+x_{2}-\widehat{W}_{1}^{T} \Psi_{1}\left(\bar{x}_{2}\right) \\
\alpha_{2}=-z_{1}-k_{2} z_{2}^{1 / 3}+u-\widehat{W}_{2}^{T} \Psi_{2}\left(\bar{x}_{2}, u\right)
\end{gathered}
$$



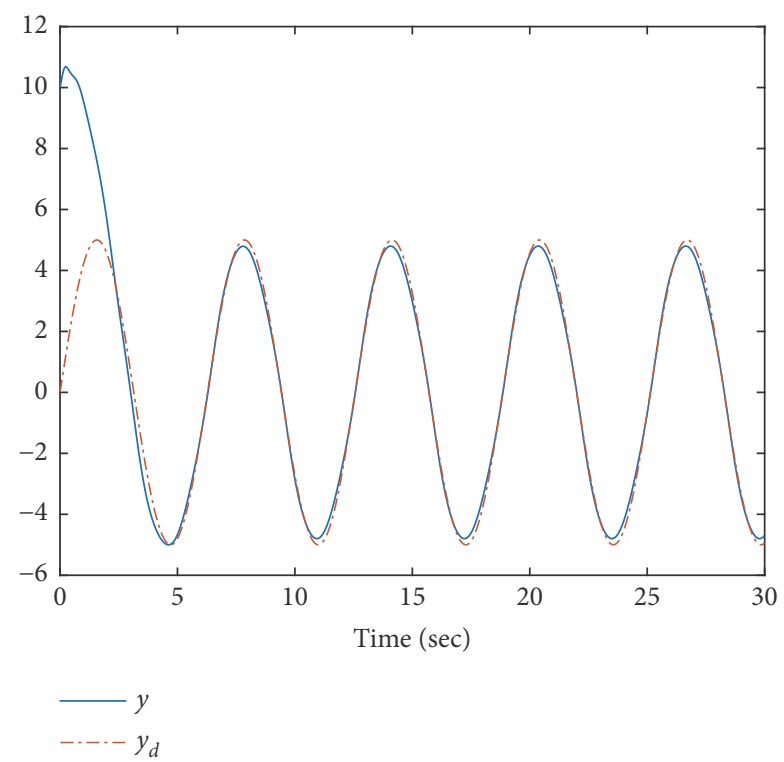

FIgURE 1: Trajectories of $y, y_{d}$.

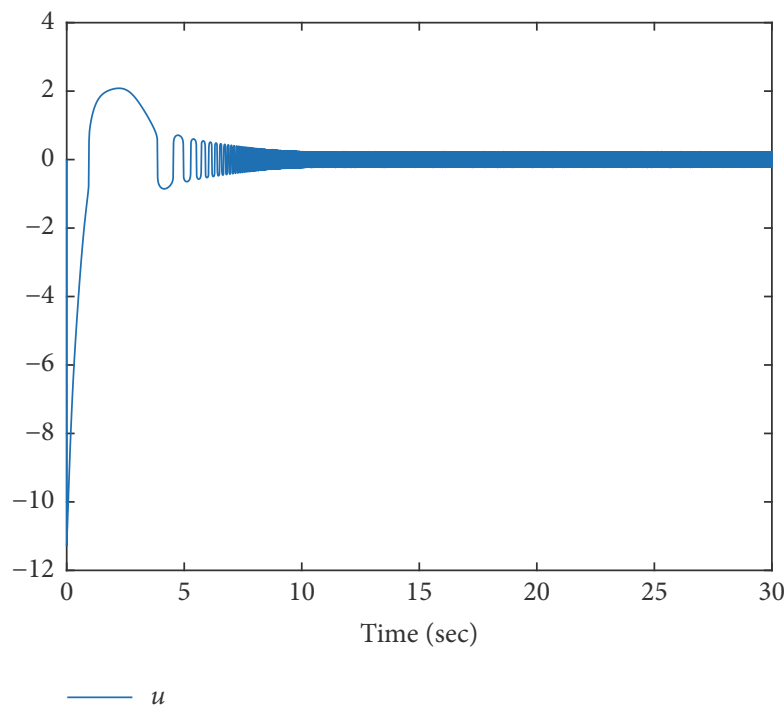

FIGURE 2: Trajectory of control input $u$.

The weight of NNs is updated by

$$
\dot{\widehat{W}}_{i}=\Gamma_{i}\left[z_{i} \Psi_{i}\left(\bar{x}_{i+1}\right)-\sigma_{i} \widehat{W}_{i}^{1 / 3}\right], \quad i=1,2,3
$$

with $z_{1}=x_{1}-y_{d}, z_{2}=x_{2}-\alpha_{1}$, and $z_{3}=u-\alpha_{2}$; the initialization of the variables is selected by $x_{1}(0)=4, x_{2}(0)=$ 4 , and $u(0)=4$.

Applying finite time NNs control method, the simulation results are shown by Figures $1-5$. Figure 1 shows the trajectory of output and desired reference trajectory. Figure 2 shows the trajectory of controller. Figures 3 and 4 show the trajectory of NNs and function which approximated by NNs. Compared with [3], the method in this paper is more generally used, and both output and weight of NNs are convergence in finite time.

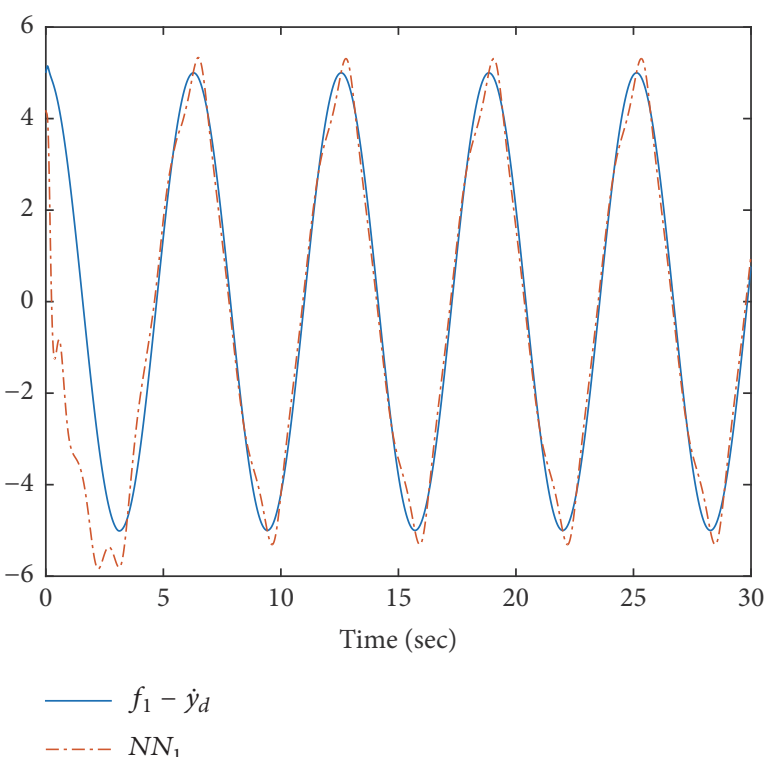

FIgURE 3: Trajectories of $f_{1}, N N_{1}$.

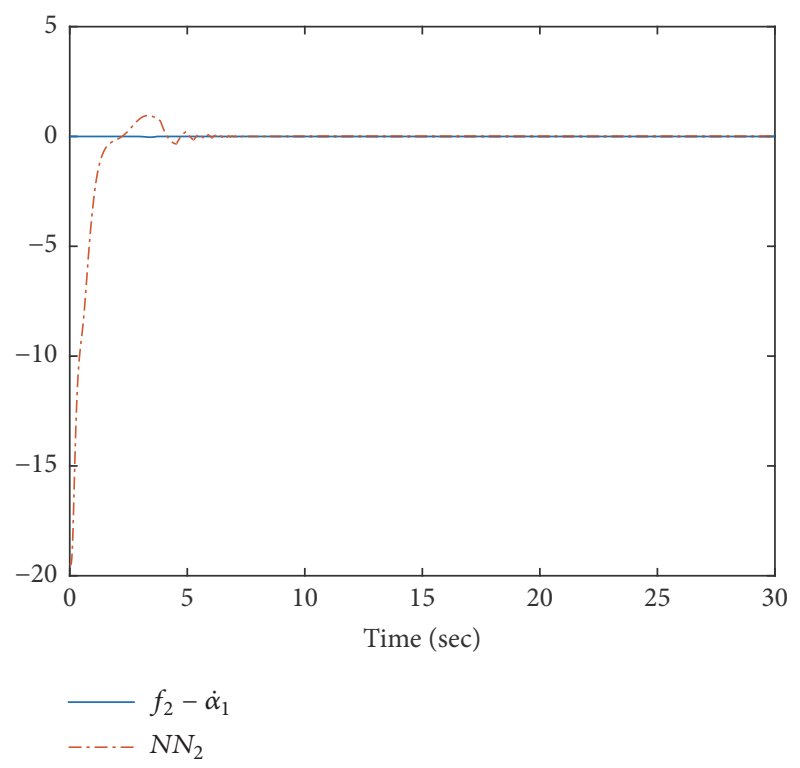

FIGURE 4: Trajectories of $f_{2}-\dot{\alpha}_{1}, N N_{2}$.

4.2. Plate-Ball Example. Consider a Plate-Ball model [43] in form of

$$
\begin{aligned}
& \left(m+\frac{I_{b}}{r_{b}^{2}}\right) \ddot{\xi}_{x}+m g u_{x}+f_{x}=0 \\
& \left(m+\frac{I_{b}}{r_{b}^{2}}\right) \ddot{\xi}_{y}+m g u_{y}+f_{y}=0
\end{aligned}
$$

where $m$ is ball mass, $I_{b}$ is ball moment of inertia, $r_{b}$ is ball radius, $u_{x}, u_{y}$ are plate inclination angle in the $x$ or $y$ direction, $f_{x}, f_{y}$ are contain the centrifugal torque resulting from rotation and other modelling error, and $y_{x d}, y_{y d}$ are ideal trajectory of $\xi_{x}, \xi_{y}$. 


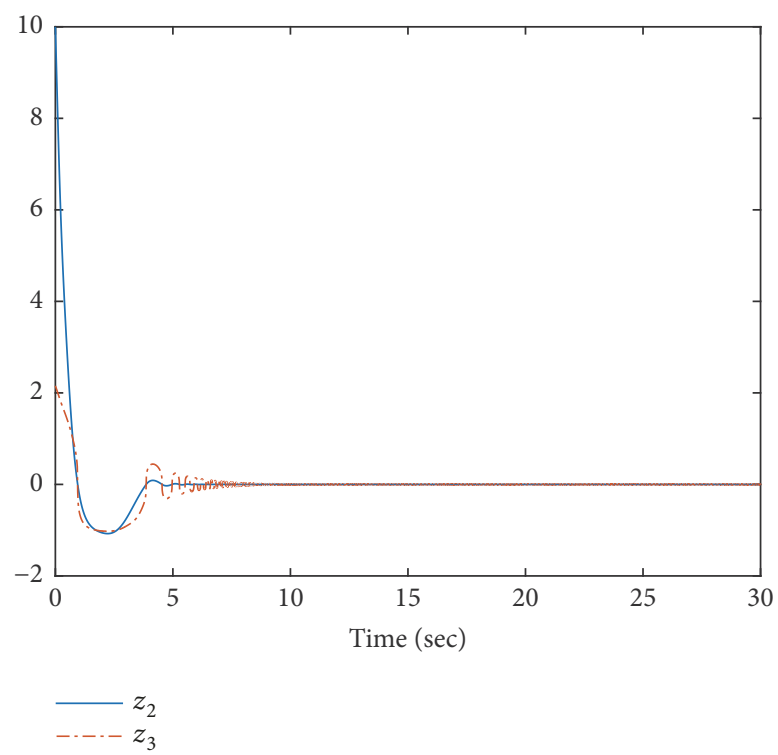

FIGURE 5: Trajectories of error system.

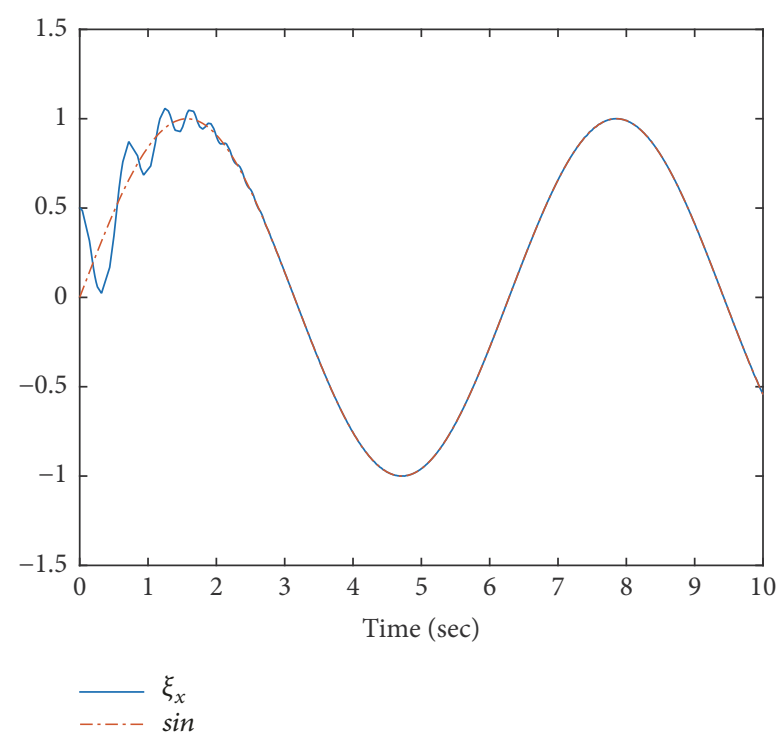

FIgURE 6: Trajectories of $\xi_{x}$ and sinusoidal signal.

Based on homeomorphism mapping technique, choose

$$
\xi_{x}-y_{x d}=r_{x} \tanh (x)
$$

then $x$ is new state variable, and therefore $\left|\xi_{x}-y_{x d}\right| \leq r_{x}$ all the time. About new state variable, we have

$$
\dot{\xi}_{x}=\dot{y}_{x d}+r_{x}\left(1-\tanh ^{2}(x)\right) \dot{x}
$$

and

$$
\ddot{\xi}_{x}=\ddot{y}_{x d}-2 r_{x} \tanh (x) \dot{x}^{2}+r_{x}\left(1-\tanh ^{2}(x)\right) \ddot{x}
$$

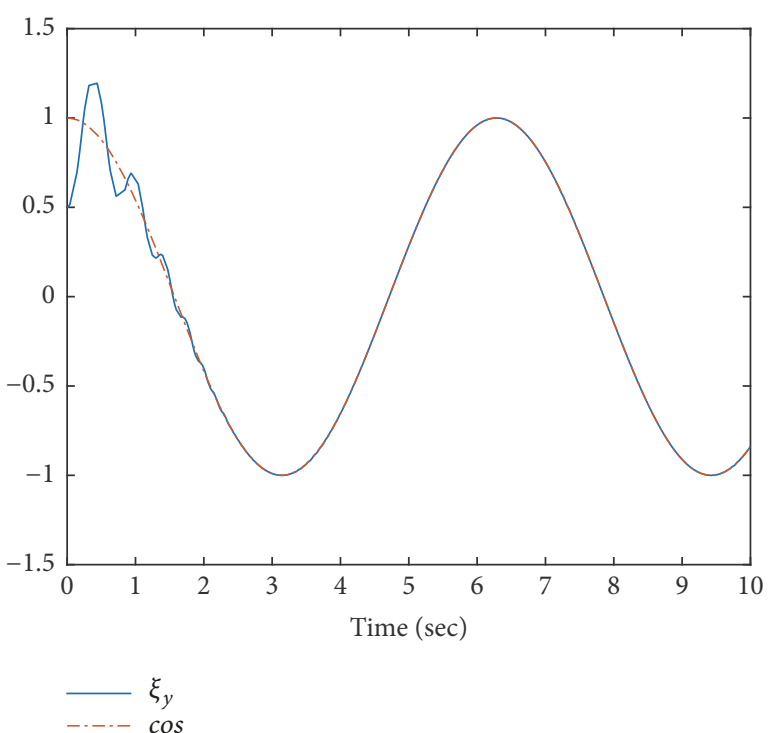

FIgURE 7: Trajectories of $\xi_{y}$ and cosine signal.

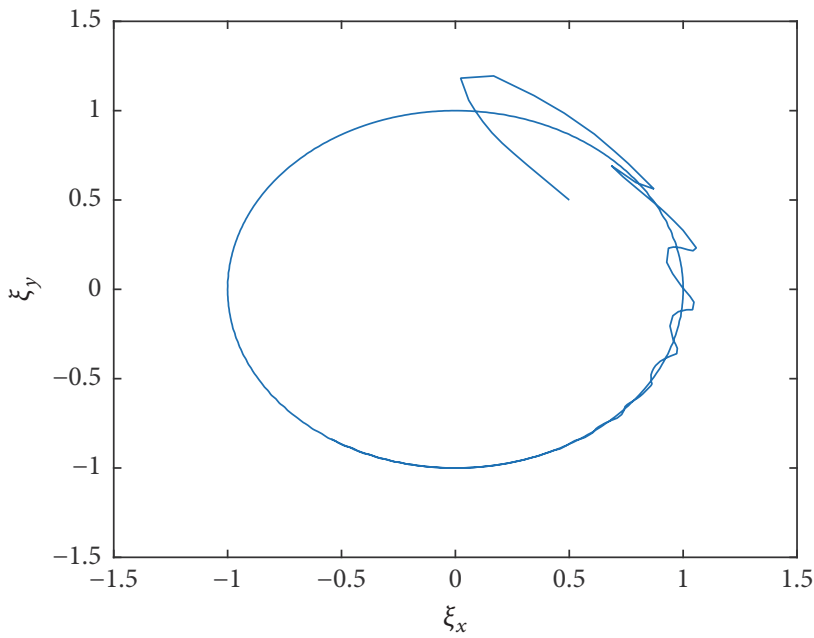

Figure 8: Trajectories of ball.

Let $x_{1}=x, x_{2}=\dot{x}$, then the above equations can be changed as

$$
\begin{aligned}
& \dot{x}_{1}=x_{2} \\
& \dot{x}_{2} \\
& =\frac{f_{x}-\left(m+I_{b} / r_{b}^{2}\right) \ddot{y}_{x d}+2 r_{x}\left(m+I_{b} / r_{b}^{2}\right) \tanh \left(x_{1}\right) x_{2}^{2}-m g u_{x}}{r_{x}\left(m+I_{b} / r_{b}^{2}\right)\left(1-\tanh ^{2}\left(x_{1}\right)\right)}
\end{aligned}
$$

then control $u_{x}$ can be designed by regular control method based on Lyapunov theory, state variable $x$ is not restricted, and $\xi_{x}$ is restricted by reference signal $y_{x d}$ and bound $r_{x}$.

Choose $f_{x}$ as unknown function, reference signal as sinusoidal signal, and NNs control. The simulation result can be obtained by Figures 6 and 7 about $\xi_{y}$ with cosine signal as reference signal. Figure 8 indicates trajectories of ball. 


\section{Conclusions}

For technique completeness, the neural networks enhanced finite time constraint control framework for a class of uncertain nonaffine pure-feedback nonlinear systems has developed analysis/proof, design, algorithms, and computational experiments. For reference of neurocontrol, this study attempts to strengthen neural network function to deal with complex nonlinear uncertain system control with different design constraints, which confirms again that the universal approximation property of neural networks feasibly effectively integrated with control system specifications. In line with further expansion of the study, some issues have been identified on the research card, such as how to design better homeomorphism mapping to realize the constraint control, how to extend the general order in finite time adaptive neural networks control, and fast computation algorithms for online computation.

\section{Data Availability}

No data were used to support this study.

\section{Conflicts of Interest}

The funding did not lead to any conflicts of interest regarding the publication of this manuscript. There are not any other possible conflicts of interest in the manuscript.

\section{Acknowledgments}

This work is partially supported by the National Nature Science Foundation of China under Grants 61273188 and 61473312 and Taishan Scholar Construction Engineering Special Funding, Shandong, China. This work is supported by Hebei Province Nature Fund Grant F2015208128 and Project in Hebei Province Department of Education QN20140157 and BJ2016020.

\section{References}

[1] W. Chen, S. S. Ge, J. Wu, and M. Gong, "Globally stable adaptive backstepping neural network control for uncertain strict-feedback systems with tracking accuracy known a priori," IEEE Transactions on Neural Networks and Learning Systems, vol. 26, no. 9, pp. 1842-1854, 2015.

[2] R. Herzallah and D. Lowe, "Distribution modeling of nonlinear inverse controllers under a Bayesian framework," IEEE Transactions on Neural Networks and Learning Systems, vol. 18, no. 1, pp. 107-114, 2007.

[3] Q. Chen, X. Ren, J. Na, and D. Zheng, "Adaptive robust finitetime neural control of uncertain PMSM servo system with nonlinear dead zone," Neural Computing and Applications, vol. 28, no. 12, pp. 3725-3736, 2017.

[4] S. S. Ge, C. C. Hang, and T. Zhang, "Stable adaptive control for nonlinear multivariable systems with a triangular control structure," Institute of Electrical and Electronics Engineers Transactions on Automatic Control, vol. 45, no. 6, pp. 1221-1225, 2000.

[5] S. S. Ge and C. Wang, "Direct adaptive NN control of a class of nonlinear systems," IEEE Transactions on Neural Networks and Learning Systems, vol. 13, no. 1, pp. 214-221, 2002.
[6] C. Hua, T. Zhang, Y. Li, and X. Guan, "Robust output feedback control for fractional order nonlinear systems with timevarying delays," IEEE/CAA Journal of Automatica Sinica, vol. 3, no. 4, pp. 477-482, 2016.

[7] X. Yang, C. Hua, J. Yan, and X. Guan, "An exact stability condition for bilateral teleoperation with delayed communication channel," IEEE Transactions on Systems, Man, and Cybernetics: Systems, vol. 46, no. 3, pp. 434-439, 2016.

[8] L. Zhang, C. Hua, and X. Guan, "Distributed output feedback consensus tracking prescribed performance control for a class of non-linear multi-agent systems with unknown disturbances," IET Control Theory \& Applications, vol. 10, no. 8, pp. 877-883, 2016.

[9] C. Hua, L. Zhang, and X. Guan, "Decentralized output feedback adaptive NN tracking control for time-delay stochastic nonlinear systems with prescribed performance," IEEE Transactions on Neural Networks and Learning Systems, vol. 26, no. 11, pp. 27492759, 2015.

[10] Y. Yang, C. Hua, and X. Guan, "Adaptive fuzzy finite-time coordination control for networked nonlinear bilateral teleoperation system," IEEE Transactions on Fuzzy Systems, vol. 22, no. 3, pp. 631-641, 2014.

[11] Q. Zhu, L. Liu, W. Zhang, and S. Li, "Control of complex nonlinear dynamic rational systems," Complexity, vol. 2018, Article ID 8953035, 12 pages, 2018.

[12] R. Herzallah and D. Lowe, "Robust control of nonlinear stochastic systems by modelling conditional distributions of control signals," Neural Computing and Applications, vol. 12, no. 2, pp. 98-108, 2003.

[13] J. Na, X. Ren, and D. Zheng, "Adaptive control for nonlinear pure-feedback systems with high-order sliding mode observer," IEEE Transactions on Neural Networks and Learning Systems, vol. 24, no. 3, pp. 370-382, 2013.

[14] S. S. Ge, F. Hong, and T. H. Lee, "Adaptive neural network control of nonlinear systems with unknown time delays," IEEE Transactions on Automatic Control, vol. 48, no. 11, pp. 20042010, 2003.

[15] K. P. Tee, S. S. Ge, and E. H. Tay, "Barrier Lyapunov functions for the control of output-constrained nonlinear systems," Automatica, vol. 45, no. 4, pp. 918-927, 2009.

[16] B. Ren, S. S. Ge, K. P. Tee, and T. H. Lee, "Adaptive neural control for output feedback nonlinear systems using a barrier lyapunov function," IEEE Transactions on Neural Networks and Learning Systems, vol. 21, no. 8, pp. 1339-1345, 2010.

[17] S. G. Nersesov and W. M. Haddad, "On the stability and control of nonlinear dynamical systems via vector Lyapunov functions," Institute of Electrical and Electronics Engineers Transactions on Automatic Control, vol. 51, no. 2, pp. 203-215, 2006.

[18] S. Wang, H. Yu, X. Gao, and W. Na, "Adaptive barrier control for nonlinear servomechanisms with friction compensation," Complexity, vol. 2018, Article ID 8925838, 10 pages, 2018.

[19] F. Cheng, L. Qu, W. Qiao, C. Wei, and L. Hao, "Fault diagnosis of wind turbine gearboxes based on DFIG stator current envelope analysis," IEEE Transactions on Sustainable Energy, pp. 1-1, 2018.

[20] J. H. Zhang, Q. M. Zhu, X. L. Wu, and Y. Li, "A generalized indirect adaptive neural networks backstepping control procedure for a class of non-affine nonlinear systems with pure-feedback prototype," Neurocomputing, vol. 121, no. 9, pp. 131-139, 2013.

[21] L. F. Zhang, Q. M. Zhu, and A. Longden, "A correlation-testbased validation procedure for identified neural networks," IEEE Transactions on Neural Networks and Learning Systems, vol. 20, no. 1, pp. 1-13, 2009. 
[22] F. Ding, L. Xu, and Q. M. Zhu, "Performance analysis of the generalised projection identification for time-varying systems," IET Control Theory \& Applications, vol. 10, no. 18, pp. 2506-2514, 2016.

[23] C. Hua, L. Zhang, and X. Guan, "Distributed adaptive neural network output tracking of leader-following high-order stochastic nonlinear multiagent systems with unknown deadzone input," IEEE Transactions on Cybernetics, vol. 47, no. 1, pp. 177-185, 2017.

[24] W. Rudin, Principles of Mathematical Analysis, McGraw-Hill, 3rd edition, 1976.

[25] Q. Chen, L. Shi, J. Na, X. Ren, and Y. Nan, "Adaptive echo state network control for a class of pure-feedback systems with input and output constraints," Neurocomputing, vol. 275, pp. 13701382, 2018.

[26] Q. Chen, S. Xie, M. Sun, and X. He, "Adaptive nonsingular fixed-time attitude stabilization of uncertain spacecraft," IEEE Transactions on Aerospace and Electronic Systems, vol. 54, no. 6, pp. 2937-2950, 2018.

[27] Y. Yang, C. Hua, and X. Guan, "Finite time control design for bilateral teleoperation system with position synchronization error constrained," IEEE Transactions on Cybernetics, vol. 46, no. 3, pp. 609-619, 2016.

[28] S.-M. Lu and D.-J. Li, "Adaptive neural network control for nonlinear hydraulic servo-system with time-varying state constraints," Complexity, vol. 2017, Article ID 6893521, 11 pages, 2017.

[29] X. Huang, W. Lin, and B. Yang, "Global finite-time stabilization of a class of uncertain nonlinear systems," Automatica, vol. 41, no. 5, pp. 881-888, 2005.

[30] Y. Hong, J. Wang, and D. Cheng, "Adaptive finite-time control of nonlinear systems with parametric uncertainty," IEEE Transactions on Automatic Control, vol. 51, no. 5, pp. 858-862, 2006.

[31] H. Wang and Q. Zhu, "Finite-time stabilization of high-order stochastic nonlinear systems in strict-feedback form," Automatica, vol. 54, pp. 284-291, 2015.

[32] C. Hua, Y. Li, H. Wang, and X. Guan, "Decentralised faulttolerant finite-time control for a class of interconnected nonlinear systems," IET Control Theory \& Applications, vol. 9, no. 16, pp. 2331-2339, 2015.

[33] D. Zhao, Q. Zhu, N. Li, and S. Li, "Synchronized control with neuro-agents for leader-follower based multiple robotic manipulators," Neurocomputing, vol. 124, pp. 149-161, 2014.

[34] D. Zhao, W. Ni, and Q. Zhu, "A framework of neural networks based consensus control for multiple robotic manipulators," Neurocomputing, vol. 140, pp. 8-18, 2014.

[35] K. Xing, P. Yang, J. Huang, Y. Wang, and Q. Zhu, "A real-time EMG pattern recognition method for virtual myoelectric hand control," Neurocomputing, vol. 136, pp. 345-355, 2014.

[36] C. Hua, C. Ge, and X. Guan, "Synchronization of chaotic lur'e systems with time delays using sampled-data control," IEEE Transactions on Neural Networks and Learning Systems, vol. 26, no. 6, pp. 1214-1221, 2015.

[37] J. Na, Y. Li, Y. Huang, G. Gao, and Q. Chen, "Output feedback control of uncertain hydraulic servo systems," IEEE Transactions on Industrial Electronics, pp. 1-1, 2019.

[38] Y.-J. Liu and S. Tong, "Barrier Lyapunov functions-based adaptive control for a class of nonlinear pure-feedback systems with full state constraints," Automatica, vol. 64, pp. 70-75, 2016.

[39] M. Z. Romdlony and B. Jayawardhana, "Stabilization with guaranteed safety using control Lyapunov-Barrier function," Automatica, vol. 66, pp. 39-47, 2016.
[40] Q. Chen, L. Tao, Y. Nan, and X. Ren, "Adaptive nonlinear sliding mode control of mechanical servo system with LuGre friction compensation," Journal of Dynamic Systems, Measurement, and Control, vol. 138, no. 2, pp. 021003-021003-9, 2016.

[41] J. Na, Q. Chen, X. Ren, and Y. Guo, "Adaptive prescribed performance motion control of servo mechanisms with friction compensation," IEEE Transactions on Industrial Electronics, vol. 61, no. 1, pp. 486-494, 2014.

[42] J. E. L. G. Hardy and G. Polya, Inequalities, Cambridge University Press, London, U.K, 2nd edition, 1989.

[43] D. Yuan and Z. Zhang, "Modelling and control scheme of the ball-plate trajectory-tracking pneumatic system with a touch screen and a rotary cylinder," IET Control Theory \& Applications, vol. 4, no. 4, pp. 573-589, 2010. 


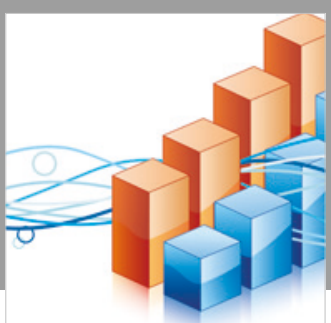

Advances in

Operations Research

\section{-n-m}
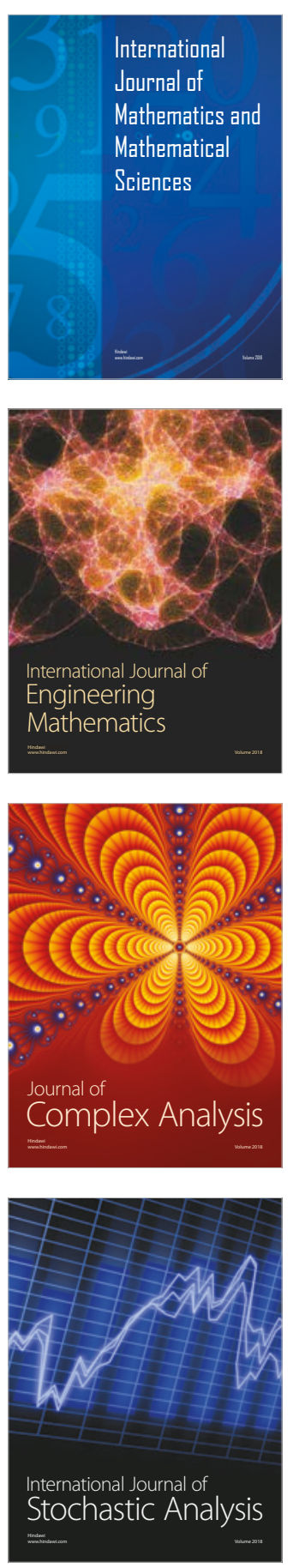
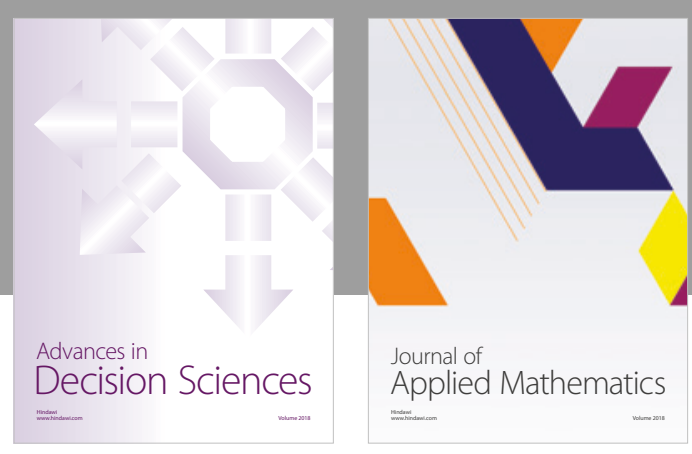

Journal of

Applied Mathematics
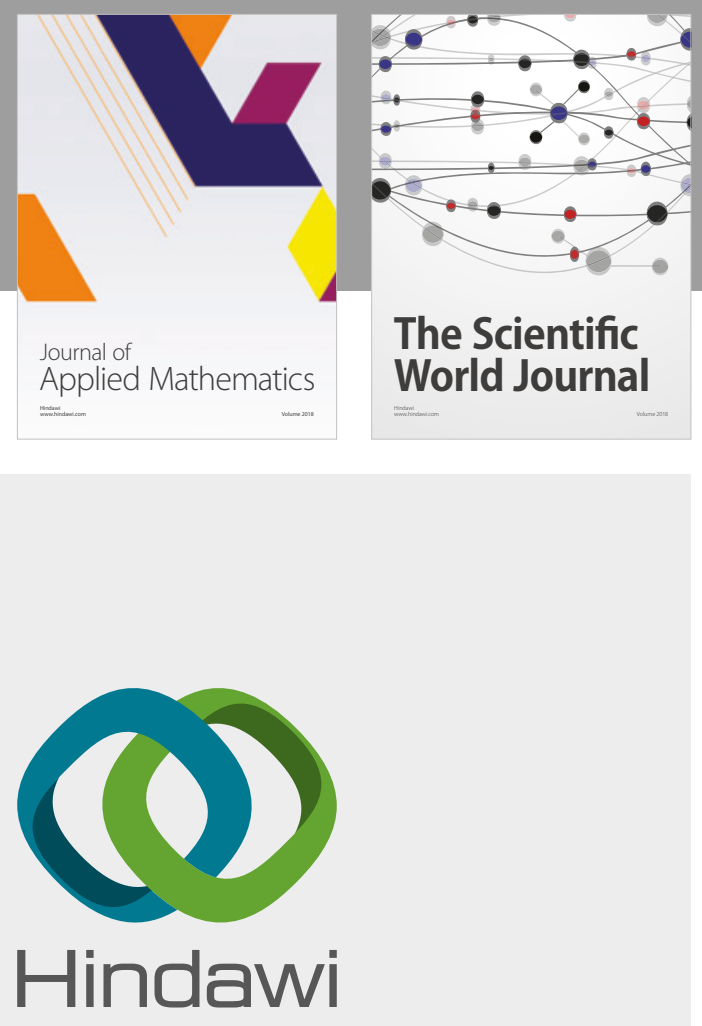

Submit your manuscripts at

www.hindawi.com

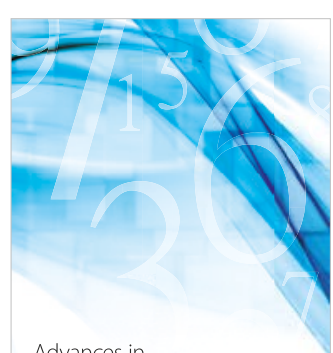

Advances in
Numerical Analysis
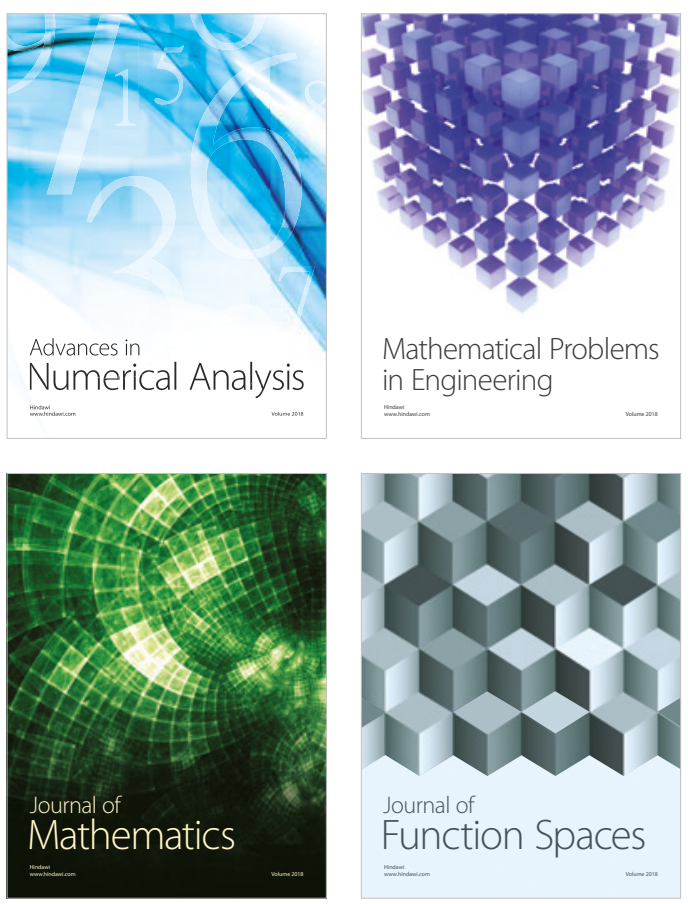

Mathematical Problems in Engineering

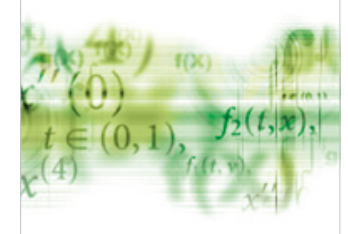

International Journal of

Differential Equations

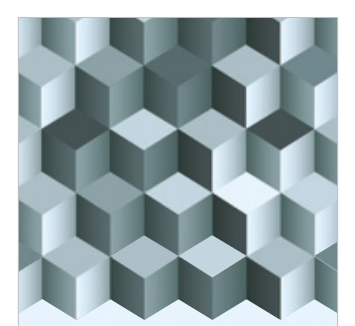

Journal of

Function Spaces
The Scientific

World Journal

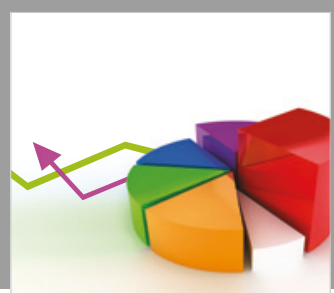

Journal of

Probability and Statistics
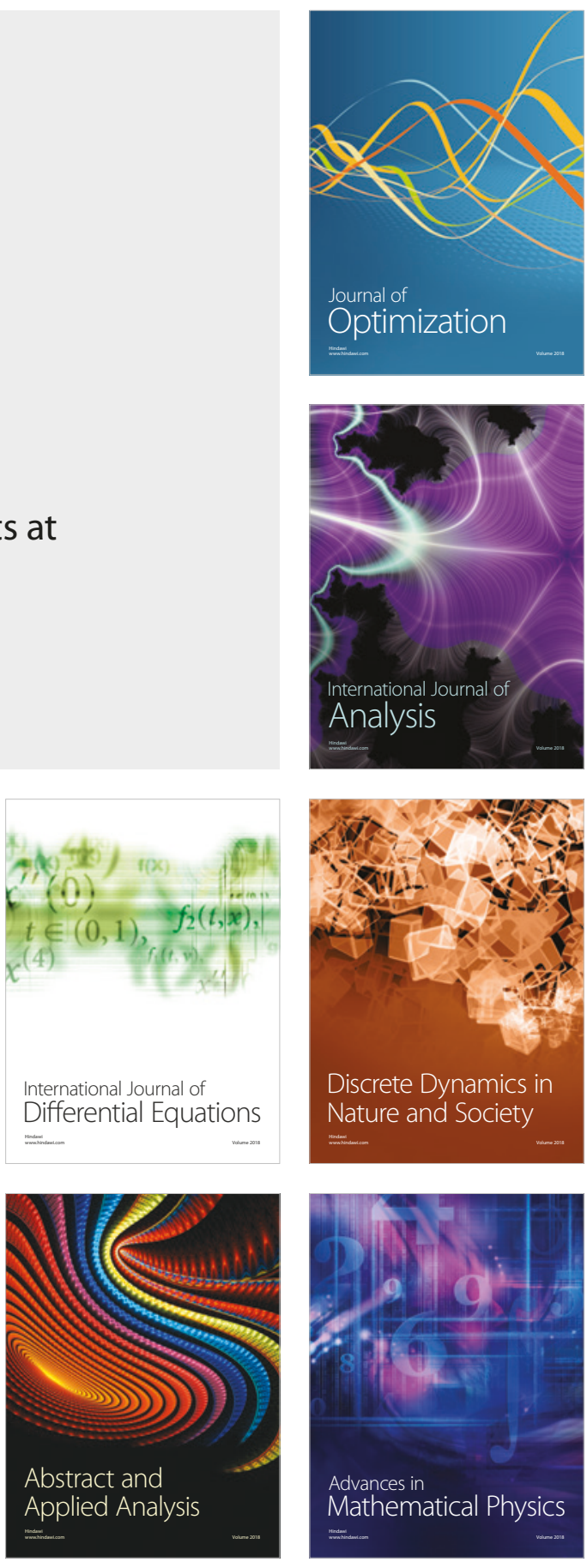